\title{
BMJ Open Service providers' perspectives, attitudes and beliefs on health services delivery for Aboriginal people receiving haemodialysis in rural Australia: a qualitative study
}

\author{
Elizabeth F Rix, ${ }^{1}$ Lesley Barclay, ${ }^{1}$ Shawn Wilson, ${ }^{1}$ Janelle Stirling, ${ }^{1}$ Allison Tong ${ }^{2,3}$
}

To cite: Rix EF, Barclay L, Wilson S, et al. Service providers' perspectives, attitudes and beliefs on health services delivery for Aboriginal people receiving haemodialysis in rural Australia: a qualitative study. BMJ Open 2013;3:e003581. doi:10.1136/bmjopen-2013003581

- Prepublication history and additional material for this paper is available online. To view these files please visit the journal online (http://dx.doi.org/10.1136/ bmjopen-2013-003581).

Received 11 July 2013 Revised 21 August 2013 Accepted 25 September 2013

\section{(1) CrossMark}

${ }^{1}$ University Centre for Rural Health, School of Public Health, The University of Sydney, Lismore, New South Wales, Australia

${ }^{2}$ The Centre for Kidney Research, The Children's Hospital at Westmead, Sydney, New South Wales, Australia

${ }^{3}$ School of Public Health, The University of Sydney, Sydney, New South Wales, Australia

\section{Correspondence to}

Elizabeth F Rix;

Elizabeth.rix@ncahs.health.

nsw.gov.au

\section{ABSTRACT}

Objective: Providing services to rural dwelling minority cultural groups with serious chronic disease is challenging due to access to care and cultural differences. This study aimed to describe service providers' perspectives on health services delivery for Aboriginal people receiving haemodialysis for end-stage kidney disease in rural Australia.

Design: Semistructured interviews, thematic analysis Setting: A health district in rural New South Wales, Australia

Participants: Using purposive sampling, 29 renal and allied service providers were recruited, including nephrologists, renal nurses, community nurses, Aboriginal health workers, social workers and managers. Six were Aboriginal and 23 non-Aboriginal. Results: Improving cultural understanding within the healthcare system was central to five themes identified: rigidity of service design (outreach, inevitable home treatment failures, pressure of system overload, limited efficacy of cultural awareness training and conflicting priorities in acute care); responding to social complexities (respecting but challenged by family obligations, assumptions about socioeconomic status and individualised care); promoting empowerment, trust and rapport (bridging gaps in cultural understanding, acknowledging the relationship between land, people and environment, and being time poor); distress at late diagnosis (lost opportunities and prioritise prevention); and contending with discrimination and racism (inherent judgement of lifestyle choices, inadequate cultural awareness, pervasive multilevel institutionalised racism and managing patient distrust).

Conclusions: Service providers believe current services are not designed to address cultural needs and Aboriginality, and that caring for Aboriginal patients receiving haemodialysis should be family focused and culturally safer. An Aboriginal-specific predialysis pathway, building staff cultural awareness and enhancing cultural safety within hospitals are the measures recommended. Increasing patient support for home haemodialysis may improve health and the quality of care outcomes.

\section{ARTICLE SUMMARY}

Strengths and limitations of this study

- We actively sought a broad range of views and experiences through purposive sampling.

- Our study included only service providers within a rural area of New South Wales, Australia

- This study recommends strategies for providing family-focussed treatment and care to rural Aboriginal renal patients that addresses cultural needs.

- Provision of culturally safer care may help to address Aboriginal people's historical distrust of mainstream health systems.

\section{INTRODUCTION}

In Australia, the incidence of end-stage kidney disease (ESKD) is eight times that of non-Indigenous populations. ${ }^{1}{ }^{2}$ Haemodialysis (HD) is the most common life-sustaining treatment for Aboriginal patients with ESKD. Health disparities, reluctance to engage with health services, miscommunication and poorer treatment outcomes have been reported in Aboriginal people receiving HD. ${ }^{3}{ }^{4}$ Similar disparities and challenges exist for Indigenous peoples of other first world nations, for example, Canada, New Zealand and the USA. $^{25}$

Australian Aboriginal people suffer higher levels of mortality and morbidity from kidney disease than non-Aboriginal Australians. ${ }^{6}$ Diabetes is a leading contributor to ESKD and is a comorbidity in the majority of patients, with frequent poor management of blood pressure and glycaemic control also being contributing factors. ${ }^{78}$ Socioeconomic disadvantage and late referral to a nephrologist are also known to increase morbidity and decrease survival time for patients with ESKD. ${ }^{9} 10$ 
Providing health services to minority cultural groups with chronic disease is challenging because of access and negotiating cultural differences. ${ }^{11}{ }^{12}$ In the Aboriginal population, further complications arise from past traumatic experiences when accessing mainstream health services. ${ }^{13} 14$ The history of colonisation and the resulting trauma experienced has perpetuated a distrust of western healthcare organisations. ${ }^{15}$ Power imbalances and a lack of understanding between service providers and Aboriginal patients still exist. ${ }^{16}$

Healthcare providers working with Aboriginal people have a responsibility to ensure cultural safety. Shortfalls in cultural safety limit Aboriginal patients' ability to access information about their disease and treatment. ${ }^{17}$ This can contribute to reduced treatment adherence and increased morbidity and mortality for people already suffering a heavier burden of chronic disease compared to non-Aboriginal Australians. ${ }^{18}{ }^{19}$ Lack of trust and rapport between clinicians and Aboriginal patients can result in patient disempowerment and suspicion towards healthcare services. ${ }^{20}$ The attitudes of healthcare providers shape how care is provided to patients and can influence health service design.

This study describes service providers' perspectives on health services delivery for Aboriginal HD recipients in a rural region of New South Wales, Australia. A better understanding of this can inform strategies to promote cultural safety and improve the quality of care.

\section{METHODS}

\section{Participant selection and setting}

Participants were recruited in a rural/regional location using a purposive selection strategy ${ }^{21}$ to increase diversity across health professionals from a range of disciplines, years of experience, age and gender. Participants were identified by consulting senior clinicians and Aboriginal health workers. There were 23 non-Aboriginal and 6 Aboriginal participants. Disciplines included in the sample were: medical, nursing (renal, community and nurse practitioner), renal case managers, hospital management, health policy, social work, Aboriginal health workers and Aboriginal liaison officers. Interviews were held at a time and place designated by participants. Written consent was obtained from all participants.

\section{Data collection}

Semistructured face-to-face interviews were conducted with service providers involved in the care of rural Aboriginal patients receiving HD. The question guide was developed informed by literature and discussion among the research team. To stimulate discussion, participants were asked to read three case studies that were adapted from interviews with Aboriginal clients of the services (see online supplementary appendix A). These were developed in consultation with senior clinicians and piloted on several renal nurses, who gave positive feedback on how these 'real-life' scenarios enabled them to focus on the challenges facing their Aboriginal patients. Based on interviews with 18 rural Aboriginal patients on HD, these case studies encompassed the key concerns identified by patients. Participants were asked to read the case studies prior to being interviewed. This helped to ensure that the interviews covered issues that were important to patients. This strategy was useful in eliciting participants' levels of cultural knowledge, attitudes and beliefs, based on 'real-life' scenarios in a nonconfronting manner. This approach accords with grounded theory where "the entire research process is interactive...we bring past interactions and current interests into our research, and we interact with our empirical materials and emerging ideas." ${ }^{22}$

Interviews were conducted between July and November 2012. Each interview averaged $1 \mathrm{~h}$, facilitated by the lead author (EFR). Interviews were digitally recorded and transcribed. Data analysis was conducted concurrently during data collection in accord with grounded theory methods. ${ }^{22}$ Theoretical saturation ${ }^{23}$ was reached at approximately 24 interviews; however, with a total of 29 of 31 invitees responding and wanting to be participants, it was considered respectful and appropriate to interview all 29.

\section{Analysis}

The first author led the analysis documenting preliminary concepts. Transcripts were entered into NVivo 10 (QSR International) for coding, searching and organisation of qualitative data. While not assuming to generate new theory with a relatively small circumscribed study in one location, the methods for this study were adapted from grounded theory. This was an appropriate methodology for beginning to develop relevant theory grounded in the participants' perspectives to inform health service delivery. ${ }^{21}$ Concepts were documented inductively, grouping similar or overlapping themes, patterns, relationships and common or divergent perspectives. ${ }^{24}$ This method followed Strauss's grounded theory 'coding paradigm', where theories are developed by interaction with the data. Coding moves from open (opening up the data to develop concepts) through to axial (the coding focuses around developing concepts) to selective coding (focusing on 1 category at a time). Explanations are discussed, reflected on and built from dense coding. ${ }^{25}$

To enhance the analytical framework and ensure rigour, interpretation of the data and emergent themes were discussed with members of the research team. Member checking was conducted by returning transcripts to participants to check for accuracy and inviting them to meet and discuss the preliminary analysis. Participants confirmed that the analysis and themes reflected their perspectives, attitudes and beliefs about service provision to Aboriginal people receiving HD. The validity and accuracy of the data analysis was thus enhanced by seeking multiple perspectives (ie, triangulation) including patient perspectives gained from a 
reference group of Aboriginal renal patients and Elders who guided the study; confirmation by discussions with participants; and involving multiple researchers from different disciplines in the analysis. ${ }^{26}$ The first author used self-reflexivity to check for potential biases from her dual clinician/researcher role, which may have influenced her decision-making throughout the study. ${ }^{26} 27$

\section{RESULTS}

Participant characteristics are provided in table 1. Of the 31 invitees, $29(93 \%)$ consented to participate in the study and two were unable to participate due to clinical commitments. We identified five themes: rigidity of service design, promoting empowerment, trust and rapport, responding to social complexities, distress at late diagnosis, and contending with discrimination and racism. Table 2 provides quotations illustrating the themes, and a thematic schema is provided in figure 1 .

\section{Rigidity of service design}

Participants felt frustrated and powerless to improve the Aboriginal patient's experience of HD due to the constraints of current service design and delivery. They believed that the service design was too rigid for the diverse needs of Aboriginal people. They expressed a desire for policymakers to implement new flexible strategies for this client group.

\section{Transport and access}

Most participants were aware of major logistical challenges for rural Aboriginal people accessing HD. Many believed that the poor transport arrangements restricted

\begin{tabular}{lcc} 
Table 1 Participant characteristics & \\
\hline Characteristics of interviewees (n, \%) & & \\
\hline Gender & 11 & $(38)$ \\
Male & 18 & $(62)$ \\
Female & 6 & $(21)$ \\
Aboriginal & 23 & $(79)$ \\
Non-aboriginal & 23 \\
Years of experience working with Aboriginal renal patients \\
$<5$ & 2 & $(7)$ \\
$6-10$ & 9 & $(31)$ \\
$11-20$ & 11 & $(38)$ \\
$>20$ & 7 & $(24)$ \\
Role & & \\
Senior management/policy & 3 & $(10)$ \\
Nephrologist/visiting medical officers & 3 & $(10)$ \\
Hospital medical officer & 1 & $(3)$ \\
Nurse unit manager & 4 & $(14)$ \\
In-centre renal nurse & 4 & $(14)$ \\
Home dialysis nurse & 2 & $(7)$ \\
Community nurse/nurse practitioner & 4 & $(14)$ \\
Social worker & 2 & $(7)$ \\
Aboriginal health worker & 4 & $(14)$ \\
Aboriginal liaison officers & 2 & $(7)$ \\
\hline & &
\end{tabular}

access and the patients' ability to attend dialysis and the other frequent appointments renal patients require. Participants described many instances of Aboriginal patients' negative outcomes resulting from poor access, for example, inability to attend podiatry appointments resulting in reduction or loss of mobility.

\section{Inevitable home treatment failures}

Although participants perceived home HD training to be performed well and tailored to individual needs, they expressed concerns regarding the lack of adequate home nursing support. They felt that a single home visit after patients were sent home with their HD machine, with follow-up some months later, was insufficient. Participants were concerned about the burden of care placed on patients' families. Some believed that lack of home support was setting Aboriginal patients up to fail on home HD, perceiving a reluctance on the part of patients or families to ask for advice or help.

\section{Pressure of system overload}

Many believed renal services were increasingly overstretched and under pressure, with insufficient resources to provide the support and culturally safe environments they felt were vital to Aboriginal patients. Some saw a lack of time to spend with individuals as culturally disrespectful of Aboriginal patients. Some perceived that system overload limited their capacity to communicate with patients regarding their $\mathrm{HD}$, or treatment problems.

\section{Limited efficacy of cultural awareness training}

Some participants who had attended the 1-day cultural awareness training felt that it reinforced differences between Aboriginal and non-Aboriginal people in a negative way, providing insufficient education to counter stereotyping and commonly held assumptions. Others felt that regular, ongoing training could be more effective in addressing current shortfalls in non-Aboriginal staffs' cultural knowledge. Aboriginal cultural awareness training is deemed mandatory for healthcare professionals within this health service; however, the majority of participants reported that they had not been given the opportunity to attend the 1-day session. While this training was deemed 'mandatory', most participants believed there were inadequate resources for sufficient training places or to backfill clinical staff to attend.

\section{Conflicting priorities in acute care}

The combination of chair-based HD with acutely ill patients in the same setting was felt to be inappropriate. Participants perceived this as confronting, particularly for Aboriginal people, many of whom have seen family members die from ESKD. Clinicians who had to juggle the demands of acute clinical care and providing cultural safety for Aboriginal patients felt conflicted and confronted. Several participants reported they were seen 
Table 2 Participant quotes table

\begin{tabular}{ll}
\hline Theme $\quad$ Illustrative quotations &
\end{tabular}

Rigidity of service design

Outreach

Inevitable home treatment failures

Pressure of system overload

Limited efficacy of cultural awareness training

Conflicting priorities in acute care

Responding to social complexities

Respecting but challenged by patients' family obligations
Obviously transport's a huge issue and not necessarily something we can have an effect on. Its difficult because most clients don't actually live here in town yet this is the only centre that's available (female, 40s)

One of the big things in particular is transport... In Queensland they use a bus...it travels all around and picks up everyone (male, 30s)

I would be aware that transport's probably the biggest issue for Aboriginal people accessing renal dialysis here (Aboriginal male, 40s)

We need someone basically full time to be able to do home visits, home assessments...but a lot of the time its hard, so if you've got people out in remote communities or towns. I mean it's so time consuming (male, 30s) I think that people going on home haemodialysis need extra support, and I can absolutely feel for the home haemo staff because you're cutting an umbilical cord and then you just sit and wait because you know they're gonna come back no matter who they are (female, 30s)

It's culturally appropriate to stay at home but it's not culturally appropriate to put all that expectation on that person and on their family members...like a punitive approach when it's not maintained, to the point of being so unwell, being in hospital and it being a failure (female, 30s)

The whole system is so busy and creaking all the time...people lacking time to spend with them is often mistaken for cultural disrespect...we don't listen to people. We don't often identify what their needs are and we don't spend time identifying problems, sorting them through, explaining things to individuals (male, 50s) It's rush-rush-rush, "let's get them on let's get them off". Um it doesn't matter which way you package that's what dialysis units are in an acute setting (female, 40s)

I don't believe you can do that as a one off because that's just a drop in the ocean, that's like saying "tick I've done that" and that's useless. There's got to be some sort of ongoing mechanism ongoing culture within the organisation that supports that ethos constantly (female, 30s)

It's interesting I watched the reactions of the people in the group I was with and I think the people that left that day with no real joy are probably feeling more polarised and could identify the start of the day with that feeling as well (female, 50s)

It's hard that balance. When you're busy and clinical, and l've always found if you spent more time with the patients you get into getting an understanding of their stuff and you can usually work out a fairly acceptable relationship with the client. But there is often no time (female, 50s)

Often the lack time that people have to spend with them is mistaken for lack of cultural disrespect and I think that the reality of it is the fact that we don't listen to people we don't often identify what their needs are and we don't spend time identifying problems, sorting them through, explaining things to individuals (male, 50s)

'OK Societal disadvantage, you know that they are starting from a more difficult place than the majority of other white or you know non-Aboriginal renal patients. When you hear that someone is Aboriginal you know that there's a really good chance that they're going to more complicated from a social point of view, that they're going to need more support and will need more assistance in walking the walk through the whole gamut that comes with having to conform to a way of being that's going to be completely foreign to how they've been used to living their lives' (female, 40s)

They put funerals and family in front of their dialysis. It's like their health comes second and I understand that because that's part of their culture but I see the consequences of it (female, 40s)

So they could actually do two days in a row and then miss a couple and if there was more flexibility in the days...we find if there is any funeral or family obligations, they will miss their dialysis in preference to that (female, 40s) 
Table 2 Continued

Theme Illustrative quotations

That's one of the biggest roots of the difficulties for Aboriginal people with dialysis is that in their reality there is no true sense of self, but it' all about community. But our health system is dependent on self-confidentiality, privacy, um reliant on health literacy. Whereas the Indigenous culture is about community ownership (female, 30s)

Making assumptions about patients' socioeconomic status

Individualised care

Promoting empowerment, trust and rapport Bridge gaps in cultural understanding

Acknowledging relationship between land, people and environment

Trust and rapport

Being time poor

Inadequate screening and diagnosis Lost opportunities

Prioritise prevention
I think it's very easy to assume they all are going to be socio or economically and transport disadvantaged and that is not actually always true and it is a trap to fall into that assumption...every case will be different (female, 50s)

If they're a low social economic group whether they be Aboriginal or non-Aboriginal there's that element of similarity because of the financial and lack of education. But it comes back to the difference is there's probably more support and encouragement for a non-Aboriginal person to participate in those renal dialysis units (Aboriginal male, 40s)

Everyone is totally individual. I assess on that basis as time goes on for each patient, regardless of who they are (male, 30s)

So, I think with supporting and just understanding and caring and being patient with the patients, because everyone's an individual and everyone's got different issues or feeling different about things at different stages (Aboriginal female, 40s) 'I actually think those challenges as for non-Aboriginal people will be very much related to their individual backgrounds' (female, 50s)

I would like to see more Aboriginal staff there. Even if it's apart from the hospital liaison person. But just someone there who they can relate to, who they can sit down and talk to, and who can relay their messages across to the non-Aboriginal staff (Aboriginal female, 60s)

They're a nice medium between the health service and their community... supporting vulnerable people and directing them, just basically giving them someone to go alongside the journey with them (female, 30s)

For me it is understanding the intricate respect of land, of people, of the environment and all those things that makes us whole as an Aboriginal person that makes an Aboriginal person who they are (Aboriginal male, 40s)

You need big windows, no one facing each other so in that whole the physical setup and environment of those units are much more culturally aware or in tune (female, 50s)

I think there's is a bit an element of lack of understanding of the past policies that were in place where Aboriginal people were taken away from hospitals removed, the stolen generation and the grief and loss that associates with that (Aboriginal male, 40s)

I guess that's where service providers need to understand that they need to build the relationship and the trust. They need to spend time with them and not just treat them as like a number (Male, 40s)

'I doubt that it is that simple as the nurse is too busy to talk to, because I have had many Aboriginal patients say to me "my God you've had a busy shift you been flat out, you haven't had time to stop". They've got insight into that' (female, 50s) They feel that they're not listened to or that they don't get the time that they need because it's always such a rush to get everyone on (female, 40s)

So we've got Aboriginal people dying of renal disease who don't even know and their GP's have not even made the diagnosis. And it may be that they're not going to GP (female, 50s)

it has to go back to primary health care, I think. You've got to diagnose and prevent (female, 40s)'

I mean there's more younger people getting kidney disease that I know of out in the communities but they're not doing anything about it (Aboriginal female staff member, 60s)

CKD screening was ruled out by the Department of Health two years ago. Not one health district been able to implement it in New South Wales because there are no resources allocated for it...So nobody's been able to implement it (female, 50s) 
Table 2 Continued

\begin{tabular}{ll}
\hline Theme & Illustrative quotations \\
\hline Contending with discrimination and racism & $\begin{array}{l}\text { I think they do see discrimination...I think it comes from something that isn't } \\
\text { just a system error. I think it is a community problem (female, 50s) } \\
\text { Sometimes I feel real empathy and compassion and understanding and some } \\
\text { days it's just like "Get over it, go and get a job you lazy little ass". I've had to } \\
\text { work, no one gives us a free ride (female, 40s) }\end{array}$ \\
$\begin{array}{l}\text { From a cultural awareness perspective I think we could all do with more } \\
\text { Inadequate cultural awareness } \\
\text { compromising patient safety }\end{array}$ & $\begin{array}{l}\text { Once they are on that machine the system has failed and the system will have } \\
\text { Pervasive multilevel institutionalised } \\
\text { racism }\end{array}$ \\
$\begin{array}{l}\text { had many opportunities for interventions. So the challenge is actually to get } \\
\text { effective reasonable pre-dialysis, pre-end stage care (female, 50s) }\end{array}$ \\
They come with pre-perceived ideas. If people have had a bad experience in \\
the past then that's it for everyone (female,40s)
\end{tabular}

by Aboriginal patients as lacking cultural respect in acute settings and felt powerless to address this.

\section{Responding to social complexities}

Participants identified differences in their clinical interactions with Aboriginal patients compared with non-Aboriginal patients, acknowledging a need to adapt their communication styles. They perceived the need to support Aboriginal patients and address different and poorly understood social and cultural complexity and family obligations, which they believed influenced treatment adherence and health outcomes.

\section{Respecting but challenged by family obligations}

Many participants understood that patients experienced conflicts between family and treatment obligations, prioritising funerals and travel to family or cultural events above treatment. There was awareness of Aboriginal patients' connectedness to family and country that identified 'who' and 'what' they are. While they respected patients' priorities, they were uncertain about how to resolve the conflict between necessary treatment and patients' family commitments. They felt unable to help patients within a system they felt did not acknowledge the priority of the family.

\section{Assumptions about socioeconomic status}

Some participants believed that the financial burden placed on individuals impacted on treatment adherence for those on limited incomes. Sharing resources with the family was culturally appropriate for Aboriginal patients, which limited money for travel or to support treatment.

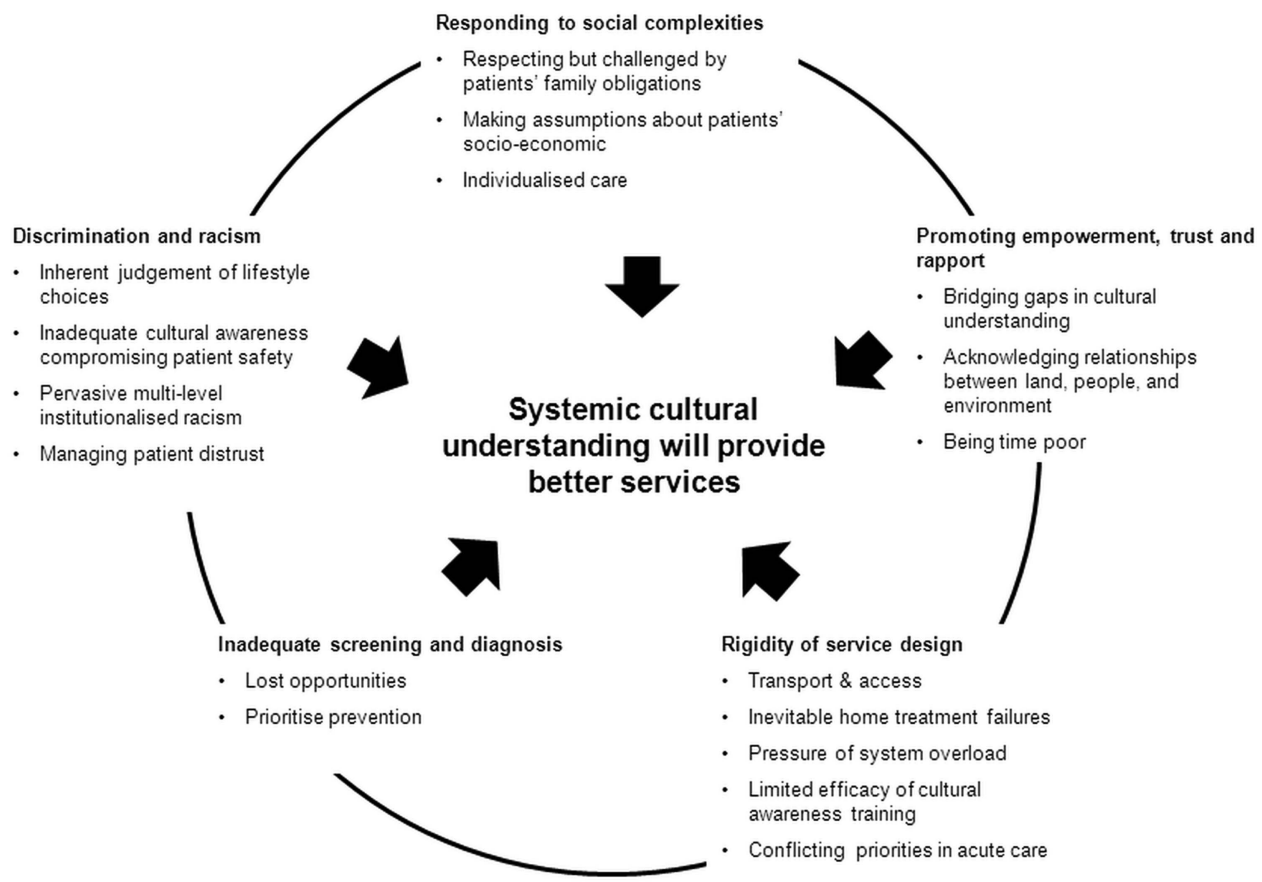

Figure 1 Thematic schema. 
Others, however, felt that it was easy to stereotype Aboriginal clients and warned that people should not make assumptions about socioeconomic status based on Aboriginality.

\section{Individualised care}

Participants believed that it was important to listen to the needs and concerns of patients in order to provide individualised care. To many this meant treating all patients as individuals, understanding their unique challenges, but emphasising the need to use simple and culturally appropriate language. They felt this was important in improving the perceived shortfalls in Aboriginal patients' understanding of the complexity of their treatment regimens.

\section{Promoting empowerment, trust and rapport}

Building trust and rapport was believed to be vital when working with Aboriginal patients. Participants were aware that being on HD creates long-term relationships between patients and clinicians, and acknowledged that when these relationships were not based on trust, disempowerment of patients often resulted. Most non-Aboriginal service providers acknowledged the need to build relationships before trust and rapport can develop. Aboriginal practitioners, however, stressed the need for a more family-focused approach. They also believed non-Aboriginal health staff should be educated about the consequences of colonisation for Aboriginal people, and understood the damaging results of patients' past negative experiences with health services.

\section{Bridging gaps in cultural understanding}

Participants felt that a designated renal-specific Aboriginal liaison or case manager, introduced at diagnosis, could provide a cultural bridge between the patients, renal staff and services. They believed that the role could encourage patients to better engage with their treatment and health services, by improving a two-way cultural understanding.

\section{Acknowledging the relationship between land, people and environment}

Most participants were aware of Aboriginal peoples' relationships to family, land and the physical environment of the places they occupy. Many desired care models that delivered a culturally safer renal service to Aboriginal people, ideally in their homes. Some suggested that simple improvements to the environment of hospital renal units, by reconfiguring the physical layout, could provide more comfortable spaces for Aboriginal people.

\section{Being time poor}

Participants working in renal units had limited time for effective communication with Aboriginal patients. They felt they lacked the ability to address the adverse effects of past traumas experienced within health services and the capacity to build Aboriginal people's trust and faith in their interactions within renal units. Some, however, believed that Aboriginal patients understood that nurses had busy workloads and would not feel ignored by their 'busyness'.

\section{Inadequate screening and diagnosis}

The majority of participants voiced their awareness of Aboriginal people frequently being diagnosed late in the progression of their disease. Aboriginal informants working with renal patients and several non-Aboriginal participants felt that this was due to Aboriginal people's inherent mistrust of mainstream health services. There were also comments about many Aboriginal people having had family members pass away or experience high levels of trauma, resulting in their avoidance of screening from a fear of being diagnosed with kidney disease themselves.

\section{Lost opportunities}

Frustration was expressed at the poor access to screening in primary healthcare settings, delaying diagnosis. Participants were distressed by lost opportunities for slowing disease progression due to late diagnosis. Some stated that inclusion of the family at diagnosis was a critical component in keeping patients engaged with predialysis strategies to slow disease progression. Participants felt that if family members understood the purpose of predialysis pathways, this might motivate them to encourage patients to participate and potentially avoid dialysis altogether. Providers believed that had impaired renal function been diagnosed earlier, premature deaths might have been avoided and that early detection of chronic kidney disease (CKD) could prevent ESKD.

\section{Prioritise prevention}

Strong concerns were voiced about the need to educate Aboriginal people regarding their risk factors for kidney disease. Aboriginal participants and some non-Aboriginal participants stressed the importance of using the family and Elders to pass on knowledge to younger generations. Some believed education strategies needed to be tailored to life experience and maturity, as these appeared to be seminal to Aboriginal peoples' understanding of their kidney disease. Others perceived that some patients would not learn until they were ready to hear about their disease.

\section{Contending with discrimination and racism}

Some participants believed that individual racism was a problem, while others considered racism to be part of the Australian 'white' culture, defining it as historical suspicion of a person of another colour or cultural background. Most agreed racism was a barrier to effective communication and their ability to develop positive relationships with Aboriginal patients. A few participants mentioned that Aboriginal people could recognise 'racist' body language, which contributed to patients' distress and insecurities. 
Inherent judgement of lifestyle choices

A minority of participants acknowledged that they sometimes made judgements about Aboriginal people's lifestyles, admitting ignorance of Aboriginal cultural values. They felt critical of Aboriginal people, who they perceived were unemployed and not helping themselves. Three of the 29 participants felt threatened by Aboriginal people and afraid of them in the clinical setting, fearing aggressive behaviour by patients when they did not understand or agree with treatment.

\section{Inadequate cultural awareness compromising patient safety}

Participants perceived insufficient cultural awareness of staff within hospitals, which translated into lack of cultural safety for Aboriginal patients. The majority viewed Aboriginal patients as victims of individualised and institutionalised racism.

\section{Pervasive multilevel institutionalised racism}

Participants believed that a health system that did not address the needs of minority cultural groups exhibited institutionalised racism. Some were distressed at the lack of an Aboriginal-specific predialysis pathway resulting in people not completing the programme. They were concerned that once diagnosed, Aboriginal people did not engage with predialysis services until their crisis admission to hospital and an emergency initiation to dialysis.

\section{Managing patient distrust}

Participants were aware of Aboriginal peoples' fear of hospitalisation. Several stated that given the history of discrimination against Aboriginal people, they are surprised that patients actually sought 'western' medical intervention. Some related patients' stories they had heard of from the past when children were taken from Aboriginal parents by welfare agencies if they attempted to access health services, or experiences of segregation and racism in hospitals. They believed that these experiences were still vividly implanted in Aboriginal people's memories, and patients still associated healthcare provision with trauma and discrimination.

\section{Inter-relation of themes}

The five themes link together under the overarching theme of 'Systemic cultural understanding will provide better services' (figure 1). Each theme relates to this major theme, with most participants acknowledging that there is a systemic lack of cultural safety impacting on service delivery and health outcomes for rural Aboriginal renal patients. A basic mistrust of mainstream services is a barrier to early screening and detection. When eventually detected, issues of transport and access to community renal nurses make it difficult to keep people at home once they start HD. When patients are forced to travel to in-centre HD, inadequate cultural awareness of staff and perceptions of pervasive institutionalised racism compound to make hospital-based treatment culturally unsafe for Aboriginal people.

\section{DISCUSSION}

Service providers caring for Aboriginal HD patients emphasised the importance of cultural safety and respect in promoting trust, rapport and patient empowerment. This involves cultural understanding and acknowledging the importance of patients' relationship to the family and country. They recognised challenges such as racism, stereotyping, social complexities within Aboriginal families, inadequate cultural awareness training and conflicting priorities of providers, patients and health services delivery modes. Service providers urged for more advocacy and flexibility for Aboriginal patients receiving HD.

Studies in remote and metropolitan settings have found that lack of cultural safety and access issues have major implications for the well-being of Aboriginal renal patients. ${ }^{4}{ }^{28-30}$ This article reports similar challenges for rural dwelling renal patients, recommending strategies to address these. A 2010 study in remote Australia demonstrated that provision of culturally appropriate services, provided by an Aboriginal-controlled medical service, has resulted in Aboriginal HD outcomes being comparable with those of non-Aboriginal Australians. ${ }^{31}$

Other studies have found culturally inappropriate healthcare, exposure to racism and poor communication $^{14} 3233$ to be among the barriers to care for Aboriginal people with chronic disease. This study, however, has identified that providing family-focused care, improving cultural awareness training and Aboriginal-specific care pathways and delivery could not only improve treatment, but also address Aboriginal people's historical distrust of mainstream health systems.

According to Taylor and Guerin, "cultural safety is achieved when the recipients of care deem care to be meeting their cultural needs." ${ }^{34}$ Cultural safety involves examining institutional structures, acknowledging assumptions, addressing power imbalances between the clinician and patients, and respecting Aboriginal cultural norms. Recent local work suggests that the current model of cultural awareness training for Australian health professionals requires improvement. ${ }^{1735}$ Cultural awareness training for healthcare providers should involve ongoing education about the priorities, values and preferences of Aboriginal patients as well as practical strategies to build trust and rapport within clinical settings.

Major barriers to access in rural areas included lack of transport to attend dialysis and frequent, multiple appointments associated with their treatment. Providing home HD was recommended by healthcare providers, but is currently limited by inadequate nursing staff. ${ }^{36}$ Home HD for remote Aboriginal patients is known to increase compliance and self-care when patients are given responsibility for their treatment, resulting in a better quality of life and outcomes. ${ }^{37} 38$ Additional in-home renal nurse support is suggested for sustaining home HD for Aboriginal patients and requires reassignment of resources and priorities. The presence of avoidable acute care episodes and late diagnosis and management suggests that urgent economic studies are 
needed to inform the costs of providing flexible, Aboriginal-focused care.

Successful treatment of chronic disease for Aboriginal people requires community consultation, local knowledge and collaboration between mainstream services and Aboriginal-controlled health services. ${ }^{32}$ The vital role of the family in Aboriginal culture was acknowledged by participants. Although they felt challenged by the social complexities relating to the family, most agreed that Aboriginal peoples' connectedness to the country and family is crucial to health and well-being. These connections are well known within Indigenous societies, where family obligations are frequently prioritised above the individual. ${ }^{39} 40$

A family-centred philosophy for Indigenous people with chronic disease, rather than an individual-based patientcentred philosophy, is recommended to address the relational nature of most Indigenous societies. The translation of knowledge down the generations by Aboriginal people, with the role of the family and Elders in passing on important information about chronic disease, is crucial. ${ }^{33}$ Participants acknowledged the impact of previous trauma experienced by Aboriginal people when encountering health services. Family-centred care may play an important role in addressing this by empowering patients and families to communicate with service providers about achieving culturally appropriate care.

Institutionalised racism was identified by healthcare providers in our study and is known to contribute to persistent health disparities in countries where Indigenous people have been colonised. ${ }^{41}$ There is a need to provide treatment and care that addresses different belief systems. ${ }^{11} 12$ Participants cited the lack of a separate Aboriginal-engaged and Aboriginal-led predialysis pathway as an example of pervasive institutionalised racism. Devising a culturally appropriate predialysis pathway, in conjunction with a renal-specific Aboriginal advocate/support person, could provide a cultural bridge. This simple and affordable strategy may assist in reducing late diagnosis and crisis until the start of HD, which is common in Aboriginal people ${ }^{39}$ and is known to increase morbidity and mortality. ${ }^{9}$ This support role may also provide education and support for those caring for a family member facing ESKD and HD. ${ }^{4}$

Healthcare providers believed that universal screening for CKD in Aboriginal people can slow disease progression and potentially avoid the need for HD. ${ }^{42}$ Further, involving the Elders and family in prevention strategies was deemed important, requiring more collaboration between renal services and Aboriginal-controlled medical services, a trusted source for screening in Aboriginal communities.

Our study includes a range of healthcare providers involved in the care of Aboriginal HD recipients, making explicit many of the challenges and barriers in providing culturally safe and respectful care. However, there are some limitations. Data were collected from one rural locality, and therefore transferability to other areas may be limited. We would, however, argue that our use of purposive sampling gave considerable strength to this study by including a diverse and broad range of perspectives and experience, thereby increasing the potential for transferability of findings and recommendations for service delivery to Aboriginal people experiencing renal disease in other rural communities. ${ }^{43}$ This study has focused on services for Aboriginal people on HD only and not included peritoneal dialysis (PD) because there were no Aboriginal patients receiving $\mathrm{PD}$ in the rural region at the time of this study. Further research exploring service delivery for Aboriginal people receiving $\mathrm{PD}$ is recommended.

\section{CONCLUSION}

Service providers believe current services are not designed to address cultural needs and Aboriginality, and that caring for Aboriginal patients receiving HD should be family-focused and culturally safer. Prioritising prevention by increased screening and education about kidney disease is paramount. An Aboriginal-specific predialysis pathway, building staff cultural awareness and enhancing cultural safety within hospitals are recommended. Increasing patient support for home HD may improve health and the quality of care outcomes. Implementing the recommendations from this study may also help in addressing patient mistrust of mainstream health services and reduce the impact of institutionalised racism on Aboriginal renal patients.

Acknowledgements The authors gratefully acknowledge the Bundjalung Nation, on whose lands this research took place. They also thank the community reference group members who provided invaluable guidance for this study.

Contributors EFR designed the study, conducted and transcribed the interviews, carried out the thematic analysis and drafted the manuscript. LB contributed to the study design, participated in the thematic analysis and critical review and edited the first and subsequent manuscript drafts. SW provided intellectual input and a critical review and also edited the first and subsequent manuscript drafts and assisted with thematic analysis. JS critically reviewed and edited the first and subsequent manuscript drafts and assisted with thematic analysis. AT contributed to the study design, participated in the thematic analysis and critically reviewed the first and subsequent manuscript drafts.

Funding EFR is supported by The Clinical Excellence Commission, Ian O'Rourke Scholarship in Patient Safety.

\section{Competing interests None.}

Ethics approval The University of Sydney Human Research Ethics Committee (HREC); The North Coast Area Health Service HREC; The Aboriginal Health and Medical Research Council of New South Wales.

Provenance and peer review Not commissioned; externally peer reviewed.

Data sharing statement No additional data are available.

Open Access This is an Open Access article distributed in accordance with the Creative Commons Attribution Non Commercial (CC BY-NC 3.0) license, which permits others to distribute, remix, adapt, build upon this work noncommercially, and license their derivative works on different terms, provided the original work is properly cited and the use is non-commercial. See: http:// creativecommons.org/licenses/by-nc/3.0/

\section{REFERENCES}

1. Australian Institute of Health and Welfare. The health and welfare of Australia's Aboriginal and Torres Strait Islander people, an overview. Canberra: AlHW, 2011. 
2. Pugsley $\mathrm{D}$, Thomas M. Renal disease in Indigenous populations. Nephrology 1998;4:S1-3.

3. Anderson K, Devitt J, Cunningham J, et al. 'All they said was my kidneys were dead': Indigenous Australian patients' understanding of their chronic kidney disease. Med J Aust 2008;189:499-503.

4. Burnette L, Kickett M. Dislocation and dialysis in Aboriginal patients with renal failure. Aboriginal Islander Health Worker J 2009;33:10-13.

5. Sood MM, Komenda P, Sood AR, et al. Adverse outcomes among Aboriginal patients receiving peritoneal dialysis. CMAJ 2010;182:1433-39.

6. Australian Institute of Health and Welfare. Chronic kidney disease in Aboriginal and Torres Strait Islander people. Canberra: AlHW, 2011.

7. Australian Department of Health and Ageing. Central Australia Renal Study. Canberra: The George Institute for Global Health, 2011.

8. Cunningham J. Socio-economic gradients in self-reported diabetes for Indigenous and non-Indigenous Australians aged 18-64. Aust $N$ Z J Public Health 2010;34:S18-24.

9. Cass A, Cunningham J, Arnold PC, et al. Delayed referral to a nephrologist: outcomes among patients who survive at least one year on dialysis. Med J Aust 2002;177:135-8.

10. Cass A, Cunningham J, Snelling P, et al. Urban disadvantage and delayed nephrology referral in Australia. Health Place 2003:9:175-82.

11. Evangelista LS, Kim Y, Kopple JD, et al. Racial/ethnic differences in illness, perceptions in minority patients undergoing maintenance hemodialysis. Nephrol Nurs J 2012;39:39-48.

12. Griva $\mathrm{K}, \mathrm{Ng} \mathrm{HJ}$, Loei $\mathrm{J}$, et al. Managing treatment for end-stage renal disease-a qualitative study exploring cultural perspectives on facilitators and barriers to treatment adherence. Psychol Health 2013;28:13-29.

13. McBain-Rigg KE, Veitch C. Cultural barriers to health care for Aboriginal and Torres Strait Islanders in Mt. Aust J Rural Health 2011;19:20-7.

14. Durey A, Wynaden D, Thompson SC, et al. Owning solutions: a collaborative model to improve quality in hospital care for Aboriginal Australians. Nurs Inq 2012;19:144-52.

15. O'Donoghue L. Towards a culture of improving Indigenous health in Australia. Aust J Rural Health 1999;7:64-9.

16. Fredericks $B$. What health services within rural communities tell us about Aboriginal people and Aboriginal health. Rural Soc $J$ 2010;20:10-20.

17. Westwood B, Westwood G. Aboriginal Cultural Awareness Training: policy v. accountability-failure in reality. Aust Health Rev 2010;34:423-9.

18. Polaschek NR. Cultural safety: a new concept in nursing people of different ethnicities. J Adv Nurs 1998;27:452-7.

19. Durey A. Reducing racism in Aboriginal health care in Australia: where does cultural education fit in? Aust N Z J Public Health 2010;34(S1):S87-92.

20. Burnette L, Kickett M. 'You are just a puppet': Australian Aborigina people's experience of disempowerment when undergoing treatment for end-stage renal disease. Ren Soc Australasia J 2009;5:113-18.

21. Richards L, Morse JM. Read me first for a user's quide to qualitative methods. 3rd edn. Thousand Oaks: Sage Publications, 2013.

22. Charmaz K. Grounded theory in the 21st century. In: Denzin NK, Lincoln YS, eds. The Sage handbook of qualtitative research. 3rd edn. Thousand Oaks: Sage Publications Inc., 2005.
23. Morse JM. The significance of saturation. Qual Health Res 1995;5:147-9.

24. Kvale S. Interviews. An introduction to qualitative research interviewing. Thousand Oaks, California: Sage, 1996.

25. Strauss. Qualitative analysis for social scientists. New York Cambridge University Press, 1987.

26. Tong A, Chapman JR, Israni A, et al. Qualitative research in organ transplantation: recent contributions in clinical care and policy. $A m \mathrm{~J}$ Transplant 2012;13:1390-9.

27. Dowling M. Approaches to reflexivity in qualitative research. Nurse Res 2006;13:7-21.

28. Bennett $\mathrm{E}$, et al. Cultural factors in dialysis and renal transplantation among Aborigines and Torres Strait Islanders in North Queensland. Aust J Public Health 1995;19:610-15.

29. Rivalland P. It's more than machines and medicine: they should understand there's a Yanangu way. Darwin: Western Desert Nganampa Walytja Palyantjaku Tjutaku Aboriginal Corporation \& CRCAH, Darwin, 2006

30. Preston-Thomas A, Cass A, O'Rourke P. Kidney disease in Aboriginal and Torres Strait Islander people Aboriginal \& Islander Health Worker Journal 2007;31:3-5.

31. Marley JV, Dent HK, Wearne M, et al. Haemodialysis outcomes of Aboriginal and Torres Strait Islander patients of remote Kimberley region origin. Med J Aust 2010;193:516-20.

32. Liaw ST, Lau P, Pyett P, et al. Successful chronic disease care for Aboriginal Australians requires cultural competence. Aust $\mathrm{NZJ}$ Public Health 2011;35:238-48.

33. Aspin C, Brown N, Jowsey T, et al. Strategic approaches to enhanced health service delivery for Aboriginal and Torres Strait Islander people with chronic illness: a qualitative study. BMC Health Serv Res 2012;12:143.

34. Taylor K, Guerin P. Health care and Indigenous Australians: cultural safety in practice. 1st edn. South Yarra: Palgrave Macmillan, 2010.

35. Franks T. Aboriginal cultural awareness training evaluation. Lismore: Clinical Education \& Training Institute, 2011:37.

36. NCAHS. Supporting home-based dialysis implementation plan 2009-2014. Lismore: North Coast Area Health Service, 2009.

37. MacGregor MS, Agar JWM, Blagg CR. Home haemodialysisinternational trends and variation. Nephrol Dial Transplant 2006;21:1934-45.

38. Villarba A, Warr K. Home haemodialysis in remote Australia. Nephrology 2004;9:S134-7.

39. Anderson K, Cunningham J, Devitt D, et al. 'Looking back to my family': Indigenous Australian patients' experience of hemodialysis. BMC Nephrol 2012;13:114.

40. Kidd J, Gibbons V, Kara E, et al. A whänau ora journey of Mäori men with chronic illness: a Te Korowai analysis. AlterNative 2013;9:126-41

41. Larson A, Gillies M, Howard PJ, et al. It's enough to make you sick: the impact of racism on the health of Aboriginal Australians. Aust $N$ Z J Public Health 2007;31:322-9.

42. NSW Health. Kidney health check: promoting the early detection \& management of chronic kidney disease. In: Health N, ed. Sydney: Department of Health, NSW, 2010.

43. Liamputtong P. Researching the vulnerable. Thousand Oaks: Sage Publications Inc., 2007. 